\title{
El sueño en ambientes extremos
}

\author{
Martínez Ruiz M. ${ }^{1}$, Martínez Galdámez ME. ${ }^{2}$
}

Sanid. mil. 2011; 67 (3): 310-316; ISSN: 1887-8571

\section{RESUMEN}

El sueño humano es sensible al medio ambiente. Las exposiciones al calor y al frío nocturno provocan reacciones en el sueño SWS (SlowWave Sleep) de ondas lentas y en el sueño REM (Rapid Eyes Movement) de movimientos oculares rápidos. La altitud y alta presión tienen efectos perjudiciales para el sueño, especialmente en individuos no aclimatados. Jet Lag es un trastorno aeronáutico del sueño que ocurre como consecuencia del cruce rápido de zonas horarias. En su entorno controlado los astronautas pueden dormir bien en ambiente de microgravedad. El presente artículo examina el conocimiento actual de los patrones de sueño humano en diferentes ambientes: exposición al calor y al frío, la altitud, la aviación y la microgravedad en el espacio.

PALABRAS CLAVE: Sueño, Calor, Frío, Altitud, Jet lag, Microgravedad espacial.

\section{Sleep under extreme environments \\ SUMMARY}

Human sleep is sensitive to the individual's environment. Heat and nocturnal cold exposures provokes reactions in SWS and REM sleep. Altitude and high pressure are deleterious to sleep, especially in non-acclimatized individuals. Jet lag is a recognized aeronautical sleep disorder that results from crossing time zones too rapidly for the circadian clock to keep pace. In their controlled environment, astronauts can sleep well in microgravity. The present article examines current knowledge of human sleep patterns under different environments: heat exposure, cold exposure, altitude, aviation and microgravity in space.

KEYWORDS: Sleep, Heat exposure, Cold exposure, Altitude, Jet lag, Space microgravity.

\section{INTRODUCCIÓN}

El sueño del ser humano es sensible al medio ambiente, de modo que los patrones de sueño varían según la exposición al calor y al frío (medicina ambiental), la altitud (medicina de montaña), el ambiente hiperbárico (medicina subacuática), el cruce rápido de zonas horarias a bordo de un avión (medicina aeronáutica) y la microgravedad en el espacio (medicina espacial) ${ }^{1}$.

El calor y el frío provocan fenómenos de regulación a corto y a largo plazo (adaptación y aclimatación), dependiendo de factores tales como el momento e intensidad de la exposición y la humedad ambiental ${ }^{1}$.

La altitud (ambiente hipobárico) altera el patrón normal de sueño, especialmente en individuos no aclimatados ${ }^{1}$. En Medicina de Montaña la altitud supone un distrés que depende principalmente de la susceptibilidad individual al desarrollo de respiración periódica durante el sueño, a la aclimatación, a la velocidad de ascenso $\mathrm{y}$ al nivel de altitud alcanzado, lo que conlleva unos efectos sobre el sueño perjudiciales, con pérdida de sueño SWS (Slow-Wave Sleep) junto a alteraciones respiratorias hipóxicas. En Medicina Aeronáutica los pasajeros y los tripulantes de avión que realizan vuelos transmeridianos sufren una trastorno circadiano del sueño denominado Jet-Lag².

${ }^{1}$ Col. Médico. Hospital Central de la Defensa «Gómez Ulla». Subdirección Médica. Madrid. España.

${ }^{2}$ Tte. Farmacéutica. Academia General del Aire. Farmacia. San Javier (Murcia). España.

Dirección para correspondencia: Mario Martínez Ruiz. Hospital Central de la Defensa «Gómez Ulla». Glorieta del Ejército, s/n. 28047 Madrid. E-mail: mmarruiz@oc.mde.es

Recibido: 12 de julio de 2010

Aceptado: 25 de noviembre de 2010
El ambiente hiperbárico, propio de la Medicina Subacuática, altera los patrones de sueño por la presión elevada, independientemente de los cambios gasométricos respiratorios; pero las alteraciones del sueño son reversibles durante la descompresión ${ }^{1}$.

En ambiente de microgravedad espacial controlado, los astronautas llegan a dormir aceptablemente bien, independientemente de la posición, mediante mecanismos de adaptación; sin embargo los astronautas experimentan también alteraciones circadianas del sueño, especialmente en vuelos prolongados ${ }^{1}$.

En este artículo revisaremos la influencia sobre el sueño de la altitud, la aviación y el espacio.

\section{SUEÑO Y TEMPERATURAS EXTREMAS}

La exposición al calor tiene dos efectos, según sea a corto o a largo plazo. A corto plazo, como sucede en las olas de calor que sufren las personas que viven en climas templados, la exposición al calor moderado antes de dormir provoca un aumento del sueño de ondas lentas (SWS) durante la noche siguiente; mientras que la exposición al calor durante la noche interrumpe el sueño, reduciendo el sueño SWS y/o el sueño de movimientos oculares rápidos REM (Rapid Eyes Movement). Por el contrario, a largo plazo, en sujetos aclimatados que viven en climas tórridos y tropicales, el calor provoca fenómenos adaptativos. Aunque las razas autóctonas, que viven en zonas de clima tropical seco, presentan un aumento generalizado del sueño SWS, tales incrementos son mayores durante la temporada de calor y con el ejercicio; mientras que el sueño REM, también aumentado, no varía de una estación a otra, ni tampoco con el ejercicio. Por el contrario, los habitantes que viven en climas tropicales húmedos no presentan un incremento ni del SWS ni del 
REM, comparados con los observados en el clima seco. De todo lo anterior se deduce que la calidad del sueño nocturno depende de si la exposición al calor ha sido durante el día o durante la noche, de su intensidad y de la humedad ambiental ${ }^{1}$.

La exposición al frío a corto plazo aumenta la inquietud durante el sueño y parece afectar principalmente al sueño REM. La exposición al frío polar provoca efectos adaptativos en el sueño REM, con un acortamiento proporcional a la intensidad del frío y a la activación de las hormonas del estrés. En sujetos aclimatados al frío, que viven en zonas polares, se demuestra una disminución generalizada del sueño SWS y del REM, hasta desaparecer en la temporada de invierno el sueño SWS, si bien durante la temporada de verano las fases de sueño SWS y REM aumentan de manera proporcional a la duración total de sueño ${ }^{1}$.

\section{SUEÑO Y ALTITUD}

La presión barométrica disminuye con la altitud. A unos $2.400 \mathrm{~m}$ la presión parcial arterial de oxígeno baja hasta el valor crítico de 60 $\mathrm{mm} \mathrm{Hg}$. Sin oxígeno suplementario, el denominado «mal de montaña» cursa con confusión y disminución del rendimiento mental y psicomotor; y a más altura se puede desarrollar edema cerebral y pulmonar. La altitud representa un reto que depende principalmente de la susceptibilidad a desarrollar una respiración periódica durante el sueño, de la aclimatación, de la velocidad de ascenso y del nivel de altitud alcanzado ${ }^{1}$.

El mal de montaña se asocia a desaturación de oxígeno durante el sueño en altas altitudes. La respiración periódica en la altitud está representada principalmente por la apnea central del sueño. El sueño es interrumpido con cada episodio apneico. En adultos, un moderado síndrome de apnea obstructiva del sueño (SAOS) al nivel del mar se convierte en una grave apnea central del sueño a unos $2.750 \mathrm{~m}$, con un gran número de apneas durante el sueño REM ${ }^{1}$.

El insomnio, como tal, aparece a altitudes a partir de los 2.000 $\mathrm{m}$, aumentando con elevaciones durante las primeras semanas de aclimatación. Los trastornos del sueño van desde una disminución de tiempo total de sueño, con numerosos despertares, a una disminución o desaparición del sueño de ondas lentas (SWS). Sin embargo, los estudios de investigación son escasos, adolecen de errores metodológicos y no son concluyentes ${ }^{1}$.

\section{SUEÑO Y AVIACIÓN}

El viaje aéreo puede afectar al sistema circadiano humano principalmente de dos modos: «trabajo por turnos» $\mathrm{y}$ «cambio de hora». Estos dos tipos de interferencias tienen en común la presencia de una disparidad entre la «hora biológica» interna y la hora ambiental externa y ambas afectan tanto a las tripulaciones como a los pasajeros del vuelo ${ }^{3}$.

El término «trabajo por turnos» se refiere al hecho de que los pilotos de líneas aéreas y los tripulantes de cabina de pasajeros (TCP) suelen realizar sus funciones en horas anormales del ciclo habitual de 24 horas, por ejemplo durante vuelos nocturnos o largos períodos de servicio. Los problemas que surgen de este tipo de conflicto no son específicos de las operaciones aéreas; sino que están asociados con el trabajo por turnos en general. En ciertos casos también se vuelven importantes para los pasajeros que hacen un viaje de negocios $^{3}$.

El segundo tipo de conflicto, denominado «cambio de hora», aparece después de realizar vuelos transmeridianos en dirección Este u Oeste, lo que implica transiciones a «zonas horarias» con importantes cambios en la hora ambiental ${ }^{3}$. Los efectos resultantes afectan específicamente al campo de la aviación, ya que las altas velocidades de los modernos aviones facilitan el recorrido de varias «zonas horarias» en unas pocas horas, velocidad que no puede ser alcanzada por el transporte de superficie ${ }^{3}$. El sujeto que realiza un viaje aéreo de larga distancia se encuentra de modo bastante repentino en una «zona horaria» en completo desajuste con sus procesos biológicos. Es lo que se conoce como «desincronosis», «desincronización», «disritmia» «discronismo transmeridiano» o síndrome/ trastorno «Jet Lag» 2,3. Aunque el término «síndrome» no sea del todo acertado, por cuanto el Jet Lag es un trastorno transitorio de ajuste circadiano y no una enfermedad, no cabe duda de que algunos viajeros experimentan diversos síntomas de «disconfort», y se observan con frecuencia claros trastornos del sueño y marcados deterioros del rendimiento, incluso a un nivel únicamente subjetivo ${ }^{2,3}$.

\section{JET LAG}

El desfase horario o Jet Lag (Jet Lag disorder, JLD) es un tipo de trastorno del sueño del ritmo circadiano (circadian rhythm sleep disorders, CRSDs) que tiene lugar al cruzar zonas horarias con demasiada rapidez ${ }^{2,4,5,6}$. Las consecuencias del Jet Lag son conocidas entre los pasajeros de vuelos transmeridianos de larga duración y consisten en somnolencia diurna, insomnio nocturno, depresión y deterioro en la concentración ${ }^{2}$. Todos estos problemas parecen deberse a un desajuste temporal entre el reloj interno circadiano (salida), normalmente sincronizado al ciclo de luz solar-oscuridad, y la hora externa local (llegada) ${ }^{2}$.

\section{Factores determinantes del Jet Lag}

\section{a) Dirección y sentido del vuelo}

Para la mayoría de nosotros resulta más fácil ajustar el reloj biológico cuando viajamos al Oeste que al Este (Fig. 1). Viajando al Oeste nos dormimos y despertamos más tarde, mientras que viajando al Este nos dormimos y despertamos más pronto. Dicha asimetría tiene su fundamento en el hecho de que el periodo natural del ritmo cotidiano, si lo comparamos con la mayoría de las funciones vitales, supera las 24 horas del día solar. De ahí que la mayoría de la gente tenga dificultad para dormir antes de su horario biológico habitual, resultándole más fácil « alargar» su día tras un vuelo hacia el Oeste que « acortarlo» en un vuelo hacia el Este. Se dice que los vuelos hacia el Este se asocian con un «cambio de avance» (advance shift), mientras que hacia el Oeste tiene lugar un «cambio de retraso» (delay shift) de la hora local $^{3,4}$.

\section{b) Duración y fatiga de vuelo}

Un vuelo de larga distancia, con un cambio en 3 - 4 zonas horarias, puede resultar casi imperceptible para el organismo; sin em- 
bargo, si el cambio es de 6 - 8 zonas horarias exige una adaptación completa y de bastante duración. Los vuelos de larga duración implican mayor fatiga de vuelo, un factor que puede influir en la clínica y evolución del Jet $\operatorname{Lag}^{2,3}$.

\section{c) Velocidad de crucero}

Los viajes que no provocan cambios de horario de más de 60-90 minutos por día, como por ejemplo los viajes en barco y los desaparecidos vuelos supersónicos comerciales (Concorde, Tupolev), entran dentro del «rango de ajuste» normal y, por lo tanto, permiten conseguir el ajuste durante el viaje. Cuando la velocidad de crucero se incrementa por encima de este rango, el sistema de tiempo circadiano se desajusta más y más respecto de la hora ambiental. Esto es lo que sucede viajando en los actuales aviones comerciales.

\section{d) Número de zonas horarias cruzadas}

El número de zonas horarias cruzadas es proporcional al grado de desalineación o desajuste del ritmo circadiano.

\section{e) Pérdida de sueño durante el vuelo}

Las condiciones ambientales de los aviones, como la baja humedad ( $5 \%$ a $35.000-40.000$ pies), hipoxia, baja presión de cabina (equivalente a 7.000 pies), movilidad reducida (riesgo de trombosis venosa profunda), deshidratación (sequedad de mucosas), ruido, vibraciones, turbulencias, incomodidad, alimentación, exposición a radiaciones ionizantes cósmicas), junto a factores psicológicos frecuentes en los pasajeros (pérdida de sueño previo al vuelo, miedo a volar, ansiedad), hacen difícil conciliar el sueño durante un vuelo ${ }^{3}$. La incomodidad es manifiesta sobre todo en asientos de clase turista y no tanto en la clase preferente o de negocios.

\section{f) Factores ambientales locales}

La principal acción de la periodicidad ambiental (luz/oscuridad) es sincronizar el sistema circadiano interno a un periodo de 24 horas. La disponibilidad de señales horarias locales, tales como la luz natural en el lugar de destino, es un factor clave para el reentrenamiento del reloj circadiano. Sin embargo, no siempre es posible garantizar la adecuada exposición a la luz solar (horario local, época del año, actividad social). La exposición a la luz artificial debe adecuarse a la fase del ciclo circadiano para permitir el correcto re-entrenamiento del reloj biológico ${ }^{2}$.

\section{g) Factores físicos individuales}

La capacidad para tolerar la desalineación o desajuste de fase circadiana expresa una variabilidad individual. Aunque los ritmos circadianos son endógenos, con oscilaciones genéticamente determinadas y con periodos cercanos a las 24 horas, cerca del $25 \%$ de las personas tras un vuelo que cruza 5-8 zonas horarias casi no siente ninguna molestia a causa del brusco cambio horario. Pero otro $20-25 \%$ de las personas se adaptan con gran dificultad o simplemente no pueden adaptarse ${ }^{3}$. La tolerancia circadiana disminuye con la edad y aumenta con la práctica deportiva.

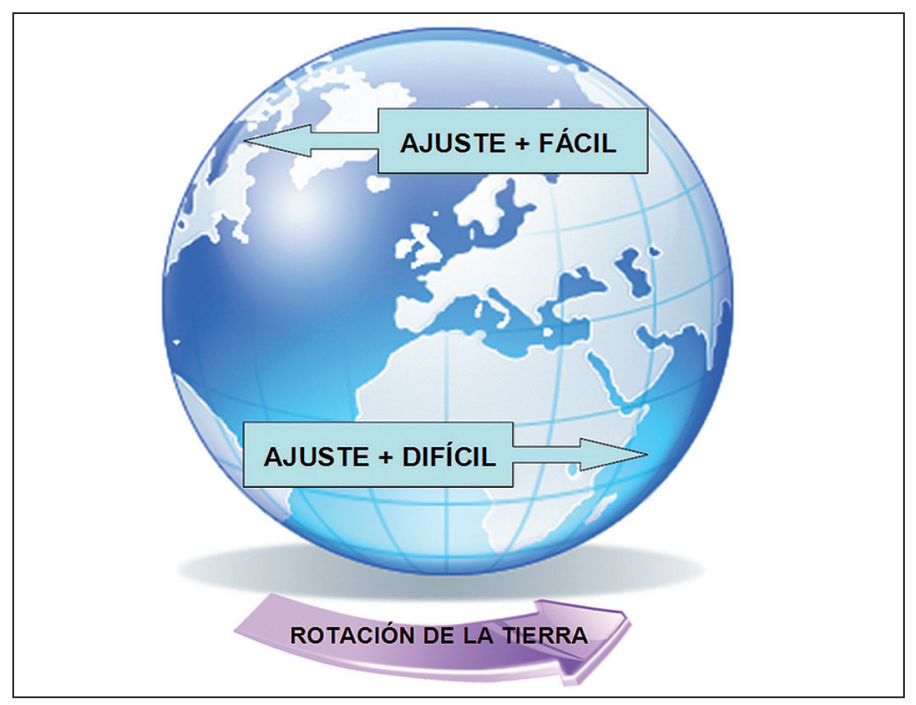

Figura 1. Sentido de rotación de la Tierra y grados de dificultad en el ajuste del reloj circadiano según el sentido del vuelo.

\section{Jet Lag y fatiga de vuelo}

En el ambiente aeronáutico el insomnio breve o transitorio, relacionado con operaciones aéreas prolongadas o nocturnas o con el Jet Lag, puede contribuir a la aparición de la fatiga de vuelo, con el riesgo final de incidentes o accidentes aéreos ${ }^{7}$. La necesidad de garantizar un sueño reparador, sin efectos que comprometan la seguridad de vuelo, llega a ser imperiosa en el medio aeronáutico, tanto para los pilotos como para los tripulantes de cabina de pasajeros $(\mathrm{TCP})^{7,8}$.

El grado de dificultad, la duración, la destreza, el conocimiento o familiaridad con la tarea, así como la motivación, el nivel de alerta (“arousal»), el estado de fatiga o descanso, los factores ambientales y la situación de la periodicidad circadiana, son factores que están relacionados con el rendimiento o performance ${ }^{7}$. Pero el rendimiento en una tarea especializada está afectado por la pérdida de sueño, sobre todo en el uso de habilidades recientemente adquiridas, más que en tareas adquiridas con anterioridad ${ }^{7}$. Dado que la tripulación de vuelo la conforman profesionales entrenados que usan habilidades adquiridas hace tiempo, a priori cabría pensar que la pérdida moderada de sueño no afectaría negativamente a sus habilidades para el vuelo; sin embargo, es posible que la fatiga de vuelo muestre algún efecto sobre la vigilancia en fases de cansancio y realización de tareas monótonas.

\section{SUEÑO Y VUELO: REGULACIONES INTERNACIONALES MÉDICO-AERONÁUTICAS}

\section{Medicamentos y vuelo}

El ambiente aeronáutico exige la máxima seguridad farmacológica, sin que se puedan o deban utilizar fármacos que presenten efectos adversos residuales, potenciales o reales, sobre el sistema nervioso central y periférico, que puedan afectar al rendimiento psicomotor (performance) o al nivel de alerta (arousal) ${ }^{7,8}$. El piloto de avión sabe que, antes de volar, necesita ciertas garantías psico-físi- 
Tabla 1. Necesidades psico-físicas de un piloto y preguntas que debe hacerse antes de volar si está tomando algún medicamento.

\begin{tabular}{lll}
\hline \multicolumn{2}{c}{ Necesidades en relación con la capacidad psico-física } & Preguntas en relación con la medicación \\
\hline Orientación espacial & $\begin{array}{l}\text { Indemnidad del sistema nervioso senso- } \\
\text { rial, visual, auditivo y propioceptivo. }\end{array}$ & ¿Me siento en condiciones de volar? \\
Capacidad mental & $\begin{array}{l}\text { Para procesar la información sensorial e } \\
\text { iniciar las medidas adecuadas de control } \\
\text { de la aeronave de forma segura. }\end{array}$ & ¿Es realmente necesario tomar la medicación? \\
& $\begin{array}{l}\text { Para llevar a cabo la misión de forma } \\
\text { decidida. }\end{array}$ & $\begin{array}{l}\text { ¿He probado este medicamento en tierra, al menos 24 ho- } \\
\text { ras antes de volar, para asegurar que no tendrá efectos ad- } \\
\text { versos sobre mi capacidad para volar? }\end{array}$ \\
\hline
\end{tabular}

$\operatorname{cas}^{9}$ y hacerse unas preguntas si está tomando algún medicamento $(\text { Tabla } 1)^{10}$.

Más del $80 \%$ de los tripulantes aéreos refieren trastornos del sueño y más de un $90 \%$ admite que la fatiga es un problema en su tipo de vuelo. Los errores se relacionan con las operaciones aéreas (sobre todo en el aterrizaje), con la fatiga de vuelo (un 10\% están relacionados) y con las horas (la zona circadiana de peligro se sitúa entre las 0 y las 6 horas). Los factores o errores humanos pueden participar hasta en un $75 \%$ de los incidentes de la aviación. Aunque los síntomas de problemas de salud menores pueden causar poco o ningún problema en tierra, pueden llegar a potenciados o ser un problema mayor en el entorno de vuelo ${ }^{10}$.

\section{Tratamiento del insomnio aeronáutico}

El consumo ocasional de un hipnótico de acción corta (por ejemplo, temazepam, zolpidem o zaleplon) o de melatonina puede ser una solución apropiada para garantizar un descanso suficiente durante una escala para un viajero ${ }^{9,10}$. Sin embargo, como la vigilancia medico-aeronáutica no siempre es posible y el lapso de tiempo suficiente entre la toma del hipnótico y el vuelo posterior no se puede garantizar (mínimo de seguridad 12 horas), el uso de cualquier hipnótico y de melatonina no está autorizado para los pilotos de aeronaves según la reglamentación aeronáutica internacional ${ }^{9}$. Es preferible recurrir y fomentar medidas no farmacológicas: no consumir cafeína, alcohol o tabaco; evitar la práctica de ejercicio antes de la hora de dormir; proporcionar un ambiente óptimo de sueño (silencio, oscuridad, aire fresco, temperatura agradable) o realizar técnicas de relajación) $)^{7,8}$.

\section{a) Tratamiento no farmacológico}

La luz es la señal de sincronización más importante del ritmo circadiano $^{2}$. La intensidad y el momento de exposición deben elegirse cuidadosamente ya que, si la exposición a la luz ocurre durante el anochecer el reloj biológico se retrasa (retrasos de fase), mientras que si la exposición a la luz se realiza por la mañana el reloj se adelanta (adelantos de fase) ${ }^{2}$. De esta manera, un pasajero puede acelerar su reentrenamiento eligiendo el momento óptimo de exposición a la luz brillante artificial, especialmente cuando no es posible la exposición solar natural ${ }^{2}$. Para viajes de hasta 8 zonas horarias de diferencia, la recomendación debe ser la exposición a la luz brillante por la mañana después de un viaje en dirección Este; y la exposición al anochecer tras un viaje en dirección al Oeste ${ }^{2}$. Los viajes de más de 8 zonas horarias de diferencia deben de ser tratados como si siempre se hubieran realizado en dirección Oeste. En estas condiciones el sistema circadiano puede inicialmente malinterpretar el amanecer como crepúsculo, y viceversa ${ }^{2}$.

El seguimiento de dietas especiales es otra estrategia de resincronización biológica. El consumo de alimentos preferiblemente proteicos durante el desayuno y el almuerzo contribuye al aumento de la producción de catecolaminas durante el día. Una cena ligera y rica en hidratos de carbonos aporta al organismo triptófano, contribuyendo a la síntesis de serotonina durante la noche. Cuando se viaja en avión es de gran trascendencia tener una alimentación equilibrada antes, durante e inmediatamente después de llegar al lugar de destino ${ }^{7}$. Por ejemplo, antes de un vuelo al Oeste se recomienda realizar una comida con alimentos que incluyan un alto contenido proteínico y un bajo contenido en hidratos de carbono; durante el vuelo no es aconsejable comer mucho, pero sí beber agua y zumos en grandes cantidades y abstenerse de consumir bebidas que contengan cafeína; tras la llegada al lugar de destino es conveniente realizar una cena ligera, con un elevado contenido en hidratos de carbono, hora u hora y media antes de acostarse ${ }^{7}$.

b) Tratamiento farmacológico

\section{Melatonina}

La melatonina, además de poseer actividad hipnótica dosis-dependiente, restablece el reloj biológico circadiano (actividad cronobiótica) y ha demostrado su eficacia en el tratamiento del Jet Lag al reducir los síntomas y acelerar la sincronización del reloj circadiano, sobre todo en los viajes en dirección Oeste. Lo habitual es que, para el tratamiento del Jet Lag, la melatonina se comience a tomar a partir del día de llegada al destino, sin que parezca que existan ventajas significativas cuando se comienza a tomar en los días previos al viaje ${ }^{2-4,7}$.

La melatonina exhibe efectos opuestos a la exposición lumínica, comportándose como una señal de oscuridad para el reloj biológico circadiano ${ }^{2}$. El momento de administración de la melatonina es crucial y debe elegirse cuidadosamente el adecuado para la sincronización circadiana: la administración de melatonina en la tardenoche adelanta el reloj biológico, mientras que la administración de melatonina por la mañana lo retrasa ${ }^{2}$. La mayor parte de los estudios 
de investigación que han evaluado la eficacia de la melatonina en el tratamiento del Jet Lag se han basado en su administración al anochecer tras un viaje en dirección al Este, lo cual parece correcto; pero este momento no es el adecuado para viajes en dirección Oeste, con 6-8 zonas horarias de cruce. En estos casos es preferible administrar una dosis baja de melatonina durante la propia noche.

La nuevas presentaciones de melatonina, comprimidos de liberación prolongada o parches transdérmicos, prolongan su concentración sérica y sus efectos. Los fármacos melatonérgicos o análogos de melatonina son nuevas opciones de tratamiento del Jet Lag. Ramelteon, Agomelatina y Tasimelteon son agonistas de los receptores de melatonina MT1 y MT2 que han demostrado su eficacia en la reducción del comienzo de la latencia del sueño y en el reajuste del ritmo circadiano de melatonina, potencial indicación del tratamiento del Jet Lag ${ }^{11}$.

\section{Hipnóticos}

Las benzodiacepinas más estudiadas en el ambiente aeronáutico han sido triazolam y temazepam, éste último no comercializado en España ${ }^{7}$. En los últimos años se han introducido paulatinamente, en el tratamiento del insomnio aeronáutico y espacial, los denominados hipnóticos no benzodiacepínicos que, aunque funcionalmente

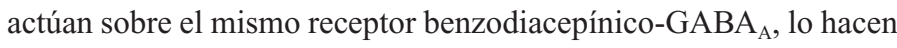
específicamente sobre subtipo 1 , son los denominados hipnóticos «zeta»: zolpidem, zopiclona y zaleplon. De estos, zolpidem y zaleplon, especialmente este último, han sido los más investigados en medicina aeroespacial ${ }^{8}$.

La decisión de utilizar agentes hipnóticos durante el viaje debe tener en cuenta sus posibles efectos adversos, como la amnesia y la confusión. A los pacientes que previamente no hayan tomado medicamentos hipnóticos se les debe aconsejar tomar una dosis de prueba en su casa antes de utilizarlos durante el viaje. Otro factor a considerar es que la inmovilidad inducida por una medicación hip-

nótica puede aumentar aún más el ya elevado riesgo de trombosis venosa profunda asociada al transporte aéreo ("síndrome de la clase turista o económica») ${ }^{12}$.

\section{Psicoestimulantes}

El aumento del consumo de cafeína puede contrarrestar la somnolencia diurna asociada con el Jet Lag. La cafeína de liberación prolongada aumenta el estado de alerta y reduce otros síntomas del Jet Lag después atravesar siete zonas horarias en vuelos hacia el Este. El riesgo principal del consumo de cafeína es la exacerbación del insomnio asociado con el Jet $\mathrm{Lag}^{2}$.

El armodafinilo, simpaticomimético central estrechamente relacionado con el modafinilo, ha demostrado mejorar la vigilia después de un viaje aéreo a través de seis zonas horarias en dirección Este, administrándose a las 07:00 horas durante 3 días consecutivos después de la llegada, consiguiendo una reducción de la somnolencia diurna y un aumento del estado de alerta, si bien hubo una mayor incidencia de cefalea, náuseas y vómitos entre los sujetos que lo consumieron ${ }^{2}$.

\section{c) Tratamiento combinado}

La utilización conjunta de luz brillante y de melatonina constituye la mejor herramienta de reajuste del reloj circadiano y se basa en los efectos inversos entre ambos: la respuesta a la exposición lumínica es mayor durante la noche, cuando la exposición solar mínima; mientras que la respuesta a la melatonina es mayor durante el día, cuando su secreción endógena es mínima². La respuesta lumínica depende de la intensidad, duración, longitud de onda, patrón y antecedentes previos de exposición; mientras que la respuesta a la melatonina es dosis-dependiente.

El tratamiento combinado se basa en los mecanismos de ajuste del reloj circadiano a la salida y en destino, que ocurren tras cruzar

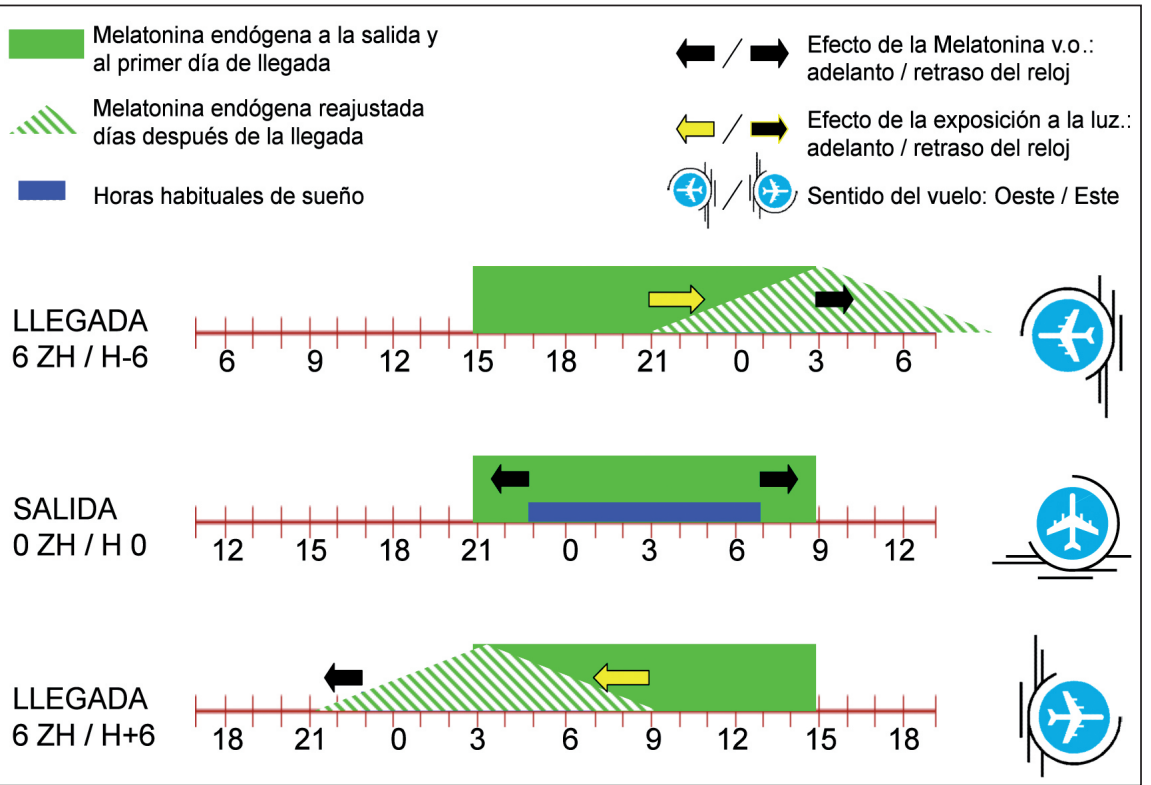

Figura 2. Cruce de zonas horarias, desajuste transitorio del reloj circadiano y reajuste facilitado por la toma de melatonina y/o por la exposición a la luz, según el sentido de vuelo. (ZH: zonas horarias. H: hora local. Explicación en el texto). diferentes zonas horarias en avión, especialmente los horarios habituales de sueño, de secreción endógena de melatonina y de exposición lumínica $^{2}$. El horario local de sueño habitual se sitúa entre las 23 y las 7 horas y la secreción de melatonina de 21 a 9 horas. En estas condiciones la exposición a la luz brillante durante la tarde y la primera parte de la noche retrasa el reloj circadiano (retraso de fase), mientras que la exposición a la luz brillante en la última parte de la noche y la mañana adelanta el reloj (avance de fase $)^{2}$. Tras un viaje en avión se debe tener en cuenta que, a la llegada al destino, y durante el primer día después, tanto el reloj circadiano como sus respuestas a la exposición a la luz y a la administración melatonina, siguen el mismo patrón de la salida, no existiendo sincronización circadiana con las nuevas horas locales de sueño/ vigilia (este desajuste entre el reloj circadiano y el horario local de sueño es la base fisiopatológica del Jet Lag) ${ }^{2}$. Durante los días sucesivos a la llegada al destino, y dada la gran sensibilidad lumínica del sistema circadiano, la exposición a 
Tabla 2. Recomendaciones para minimizar los sintomas del Jet Lag y de la fatiga de vuelo. (Los números en negrita indican la dosis habitual. ZH: zonas horarias).

\begin{tabular}{|c|c|c|}
\hline ESTRATEGIA & DIRECCIÓN OESTE & DIRECCIÓN ESTE \\
\hline \multicolumn{3}{|c|}{ ANTES DEL VIAJE } \\
\hline Ajustar reloj biológico & $\begin{array}{l}\text { Acostarse } 1-2 \mathrm{~h} \text { más tarde. } \\
\text { Exposición luz noche. }\end{array}$ & $\begin{array}{l}\text { Acostarse } 1-2 \text { h antes. } \\
\text { Exposición luz mañana. }\end{array}$ \\
\hline Sueño & \multicolumn{2}{|c|}{$\begin{array}{l}\text { 1. No dejar los preparativos del vuelo para el último minuto. } \\
\text { 2. Elegir un horario de vuelo que no altere el sueño. }\end{array}$} \\
\hline \multicolumn{3}{|c|}{ A LA LLEGADA } \\
\hline Siesta & \multicolumn{2}{|c|}{ Dormir siesta cortas (30 minutos) los primeros días. } \\
\hline Melatonina & $\begin{array}{l}\text { Dosis bajas (1-3 mg) durante la } 2^{\text {a }} \text { mitad de } \\
\text { la noche, hasta adaptarse a la hora local. }\end{array}$ & $\begin{array}{l}\text { Dosis medias (3-5 mg) a la hora local de } \\
\text { dormir, hasta adaptarse a la hora local. }\end{array}$ \\
\hline Luz $<8 \mathrm{ZH}$ de cruce & Exposición por la noche & Exposición por la mañana \\
\hline Luz $\geq 8 \mathrm{ZH}$ de cruce & $\begin{array}{l}\text { Evitarla 2-3 horas antes del anochecer los } \\
\text { primeros } 2 \text { días y, a partir del } 3^{\circ} \text {, buscar la } \\
\text { exposición por la noche. }\end{array}$ & $\begin{array}{l}\text { Evitarla 2-3 horas después del amanecer } \\
\text { los primeros } 2 \text { días, y a partir del } 3^{\circ} \text {, bus- } \\
\text { car la exposición por la mañana. }\end{array}$ \\
\hline Cafeína & \multicolumn{2}{|c|}{ Beber bebidas con cafeína por la mañana; pero evitar su consumo a partir del mediodía. } \\
\hline Hipnóticos & \multicolumn{2}{|c|}{ Valorar zolpidem 5-10 mg o zaleplon 5-10 mg al acostarse durante los primeros días. } \\
\hline \multicolumn{3}{|c|}{ DURANTE EL VUELO } \\
\hline Confort & \multicolumn{2}{|l|}{ Tratar de viajar en clase superior. } \\
\hline Bebidas & \multicolumn{2}{|c|}{$\begin{array}{l}\text { 1. Beber suficiente agua para mantenerse hidratado. } \\
\text { 2. No beber café si quiere dormir. } \\
\text { 3. No beber alcohol si se va a tomar un hipnótico. }\end{array}$} \\
\hline Hipnóticos & \multicolumn{2}{|c|}{$\begin{array}{l}\text { 1. Valorar zolpidem } 5-10 \mathrm{mg} \text { o zaleplon } 5-10 \mathrm{mg} \text {. } \\
\text { 2. No tomar si existe riesgo de trombosis venosa profunda. } \\
\text { 3. No combinar hipnótico con alcohol. }\end{array}$} \\
\hline $\begin{array}{l}\text { Prevenir la trombosis venosa pro- } \\
\text { funda }\end{array}$ & \multicolumn{2}{|c|}{$\begin{array}{l}\text { 1. Cambiar de posición con frecuencia. } \\
\text { 2. Hacer ejercicios con los pies. } \\
\text { 3. Caminar, si es posible. } \\
\text { 4. Valorar medias elásticas, dosis preventiva de Aspirina. }\end{array}$} \\
\hline
\end{tabular}

la luz solar provocará cambios de fase encaminados reajustar el reloj biológico a la nueva hora local, sincronizándose los patrones de respuesta a la luz y a la melatonina (Fig. 2) ${ }^{2}$.

\section{SUEÑO Y VUELOS ESPACIALES}

Los astronautas suelen tener dificultad para dormir durante el vuelo espacial, lo que parece deberse a una combinación de factores tales como la novedad y la emoción del vuelo espacial, la microgravedad, la cinetosis, las características de la misión, el estrés, la fatiga, el ruido ambiental, el reducido espacio de la nave y la ausencia de ciclos circadianos habituales (la luz del sol y el calor entran por la ventana de la cabina cada 90 minutos) ${ }^{13}$. Los astronautas viven la experiencia de 16 puestas de sol cada 24 horas y un nuevo día aproximadamente cada 90 minutos. En este ambiente, la duración media del sueño de los astronautas es de 6-6,5 horas, existiendo más despertares y menos sueño SWS durante la tercera parte final del sueño. Tras el regreso a la Tierra el sueño REM experimenta un aumento significativo ${ }^{13}$.

La «mejora» del sueño, que algunos astronautas refieren, puede deberse a que los efectos de la gravedad cero reducen los ronquidos, al disminuirse la resistencia de las vías respiratorias superiores durante el sueño ${ }^{14}$. 
La interrupción del sueño puede conducir a fatiga y a disminución del rendimiento de los astronautas. Para mejorar la calidad del sueño muchos astronautas han tomado hipnóticos (temazepam, zaleplon, melatonina). De hecho el 45 por ciento de todos los medicamentos utilizados por la tripulación del transbordador espacial son hipnóticos ${ }^{7,8,13}$.

\section{PAUTAS DE TRATAMIENTO RECOMENDADAS PARA EL JET LAG}

1. Un pasajero de avión va a realizar un vuelo en dirección Este, viaje ida y vuelta, cruzando 6 zonas horarias $(\mathrm{ZH})$. Las recomendaciones médico-aeronáuticas serían (Tabla 1 y Fig. 2):

a) Antes de la salida (0 ZH), adelantar progresivamente el horario de sueño 2 horas y exponerse a la luz brillante al despertarse.

b) Al llegar al destino (+ $6 \mathrm{ZH}$, dirección Este), caminar con la luz brillante solar, beber una bebida con cafeína cada mañana y tomar melatonina a una dosis media $(3 \mathrm{mg})$ a la hora de acostarse, durante 3 a 4 días, con el fin de acelerar el desplazamiento de fase a la nueva situación circadiana. Si la melatonina no bastara para facilitar el sueño, podría asociarse una dosis habitual de zolpidem (5 mg) o zaleplon (10 mg).

c) A su regreso (- $6 \mathrm{ZH}$, dirección Oeste), exponerse a la luz brillante durante la noche y tomar una pequeña dosis de la melatonina (1 mg) si se despierta antes de la madrugada.

2. Si, por el contrario, el pasajero fuera a volar en dirección Oeste, viaje ida y vuelta, cruzando $6 \mathrm{ZH}$, las recomendaciones medico-aeronáuticas serían (Tabla 1 y Fig. 2):

a) Antes de la salida $(0 \mathrm{ZH})$, retrasar progresivamente el horario de sueño 2 horas y exponerse a la luz brillante al acostarse.

b) A llegar al destino (- $6 \mathrm{ZH}$, dirección Oeste), exponerse a la luz brillante durante la noche y tomar una pequeña dosis de la melatonina ( $1 \mathrm{mg}$ ) si se despierta antes de la madrugada.

c) A su regreso (+ $6 \mathrm{ZH}$, dirección Este), caminar con la luz brillante solar, beber una bebida con cafeína cada mañana y tomar melatonina a una dosis media $(3 \mathrm{mg})$ a la hora de acostarse, durante 3 a 4 días, con el fin de acelerar el desplazamiento de fase a la nueva situación circadiana. Si la melatonina no bastara para facilitar el sueño, podría asociarse una dosis habitual de zolpidem $(5 \mathrm{mg})$ o zaleplon (10 mg).

\section{BIBLIOGRAFÍA}

1. Buguet A. Sleep under extreme environments: effects of heat and cold exposure, altitude, hyperbaric pressure and microgravity in space. J Neurol Sci 2007; 262 (1-2): 145-152.

2. Sack RL. Jet Lag. N Engl J Med 2010; 362(5): 440-447.

3. Wegmann H. Jet Lag y sueño. En: Avances en la investigación del sueño y sus trastornos. Gualberto Buela-Casal y José Francisco Navarro Humanes (Eds.) Siglo Veintiuno de España Editores, S.A 1990. ISBN: 84-323-0677-0. Págs. 139-152.

4. Sack RL; Auckley D; Auger RR; Carskadon MA; Wright KP; Vitiello MV; Zhdanova IV. Circadian rhythm sleep disorders: Part I, basic principles, shift work and Jet Lag disorders. Sleep 2007; 30(11): 1460-1483.

5. Sack R; Auckley D; Auger RR; Carskadon MA; Wright KP; Vitiello MV; Zhdanova IV. Circadian rhythm sleep disorders: Part II, advanced sleep phase disorder, delayed sleep phase disorder, free-running disorder, and irregular sleep-wake rhythm. Sleep 2007; 30(11): 1484-1501.

6. Morgenthaler TI; Lee-Chiong T; Alessi C; Friedman L; Aurora N; Boehlecke B; Brown T; Chesson AL; Kapur V; Maganti R; Owens J; Pancer J; Swick TJ; Zak R; Standards of Practice Committee of the AASM. Practice Parameters for the Clinical Evaluation and Treatment of Circadian Rhythm Sleep Disorders. Sleep 2007; 30(11): 1445-1459.

7. Martínez Ruiz M. Tratamiento del insomnio en Medicina Aeronáutica. Med Mil (Esp) 2003; 59(4): 11-22.

8. Martínez Ruiz M, Ríos F, Esteban B. Seguridad y utilidad de zaleplon en operaciones aéreas. Med Mil (Esp) 2005; 61(1): 10-14.

9. The Joint Aviation Authorities (JAA) Committee. JAA Manual of Civil Aviation Medicine. Chap. 19. Pages:19-313 to 19-326. Amendment 6. Stage 01.06.07. Global Engineering Documents, Englewood, Colorado (USA). 1 June 2009.

10. The UK Civil Aviation Authority. Information Circular (AIC) 99/2004 (Pink 72). Medication, alcohol and flying. Aeronautical. National Air Traffic Services Ltd. Aeronautical Information Service. Control Tower Building, London Heathrow Airport (UK). 14 October 2004

11. Ferguson SA, Rajaratnam SM, Dawson D. Melatonin agonists and insomnia. Expert Rev Neurother. 2010; 10(2):305-318.

12. Martínez Ruiz M, Martínez-Galdámez ME. Prevención de síndrome de la clase turista o de la trombosis del viajero. Med Mil (Esp) 2006; 62(4): 225-229.

13. Martínez Ruiz M. Retos de la Medicina Espacial en el siglo XXI. Med Aeroesp Ambient 2007; 5(2): 91-97.

14. Shi SJ, Garcia KM, Meck JV. Temazepam, but not zolpidem, causes orthostatic hypotension in astronauts after spaceflight. J Cardiovasc Pharmacol 2003; 41(1):31-39. 